\title{
Lo arbitrario del lenguaje y el lugar de la cultura ${ }^{1}$
}

Carlos R. Luis*

\begin{abstract}
El idioma es un ordenamiento efic az de esa enigm tica abundancia del mundo. Lo que nombramos sustantivo no es sino abreviatura de adjetivos y de su falaz probabilidad.
\end{abstract}

J . L Borges, Examen de met foras

Resumen: Seinvestigauna línea de pensamiento, partiendo del materialismo antiguo (Epicuro) y en su desarrollo posterior por la filosofía del I luminismo (Locke, Condillac): el postulado de un origen "natural" parael lenguaje (corporal, de respuesta al ambiente) y una evolución que lo refunda como social y por tanto arbitrario. Se analiza la idea de delimitación del mundo natural como primera condición para la supervivencia, es decir, para la producción de bienes. A parece así la cultura, que desde temprano es asociada con cultivo. Sus delimitaciones son sancionadas por el lenguaje a través de los nombres, que no designan sustancias, sino agrupamientosarbitrarios de cualidades. Losnombresson sólo formas diferenciadoras, variables, tanto como son variables las impresiones sensoriales de individuo a individuo; es la cultura lo quevienea garantizar el acuerdo, a producir el efecto de uniformidad delas designaciones. El lenguajees, entonces, un modo de nombrar un mundo clasificado de una cierta manera para producir predicaciones estables entre quienes comparten esas clasificaciones, entre quienes comparten una cultura.

Palabras clave: cultura; filosofía del lenguaje; semiótica; lingüística; comunicación.

Abstract: Thisisthestudy of a line of thought rooted in ancient materialism (Epicure) and its further development through thephilosophy of Enlightenment (Locke, Condillac): thestating of a "natural" origin for language (as a corporal response to the environment) and, later on, a redefinition of that original basisas being social, and therefore, arbitrary. Theidea of delimitation of thenatural world isintroduced as the prime condition for survival, i.e., for the production of goods. C ulture is then associated to the idea of cultivati on. I ts del imitations are sanctioned by language by means of names, which do not refer to substances, but to arbitrary collections of qualities. N amesare only differentiating, variableforms, just as sensorial impressionsare variablefrom an individual to another. C ultureworks as a guarantee of agreement, producing a uniform effect for designations. Language is then a means of naming a world which is classified in a certain way so as to allow stable predications among those who share such classifications, thussharing a culture.

Key words: culture; philosophy of language; semiotics; linguistics; communication.

* Instituto de Lingüistica, Facultad de Filosofía y Letras, Universidad de Buenos Aires. cluis@ filo.uba.ar

1. El texto original de este trabajo fue mejorado gracias a valiosas observaciones y atinados comentarios de Damián Grimozzi. 
En un principio, no fue deliberadamente(thesei) como se dio nombrea las cosas, sino quelasnatural ezas deloshombres, según sus diferentes naciones (ethne), tenían experiencias (pathe) particulares y recibían impresiones (phantasmata) particulares; así, cadanación a su manera emitíasonidosformados deacuerdo con esas experiencias y esas impresiones, conforme a las diferencias que producían los lugares de su habitat. Y sólo más tarde, por consentimiento común decada nación (ethne), fueron dados deliberadamente nombres específicos para que las designacionesfuesen menos ambiguasy másconcisas. Algunas veces los hombres introdujeron cosas desconocidas antes y sonidos que las designaban, ya formados por restricciones naturales, ya elegidos por razonamiento, según el modo dominantedeformación delas palabras; aś las designacionesse hicieron claras. [Epicuro (341-270), Carta a H eródoto, 75-76].

Elijo este pasaje para comenzar señalando la antigüedad de una relación: la de las lenguas con las etnias o naciones, con las diferencias de hábitat, con el trabajo diferenciado sobre las restricciones fonatorias. $Y$ señalo también el deliberadamente, con que se ha traducido ${ }^{2}$ la expresión griega thesei, "por convención", opuesta a "por naturaleza". El pasaje sorprende si se lo compara con las versiones de un origen verdadero de la palabra, cuando esta revelaba el ser de la cosa; como las que encontramos en las etimologías del Sócrates platónico, proferidas en una especie de transporte místico. ${ }^{3}$ El materialista Epicuro no podía menos que contrastar con la visión platónica y es así como nos ha entregado ésta, diferente, histórica, del origen y división de las lenguas. Cuando digo histórica pienso en cultural, porque lo que me propongo es mostrar que la idea de cultura contiene como un componente básico el gesto de delimitar: parcelar, diferenciar, diferenciarse; y creo que aun en su brevedad, el fragmento epicúreo ya nos dice que las lenguas son desde el inicio constructoras, 0 al menos reveladoras, de esas diferencias: étnicas, fónicas, ambientales.

En el pasaje de la Carta hay también la idea de un perfeccionamiento de las lenguas para tornar los nombres menos ambiguos y breves; para llegar a las "designaciones claras". Pensemos que en las etimologías platónicas, por el contrario, el trabajo humano fue ocultando aquella verdad prístina (de ahí la necesidad de un estado de trance para recuperarla). Es decir, hay una explicación del lenguaje ordinario por corrupción. Esto nos remite a un dato más cercano 0 familiar en nuestras culturas: la lengua adánica, 0 aquella en la que, hasta

2. Uso la traducción al francés de Paul Nizan (1965), que retoqué levemente después de cotejarla con el original griego.

3. Platón, Cratilo 396 a-e. 
Babel, D ios había hablado a los hombres. Esta visión mítico-teológica ve la acción humana sobre las lenguas no en función de un afinamiento de la comunicación, como en Epicuro, sino como la causa de supuestas imperfecciones. Se explica aś la diferencia entre lenguas por medio de un castigo a cierta conducta de los hombres. La distinción, la diversidad de las lenguas es atribuida a un concepto negativo: la confusión, que provoca el desorden de la multiplicación y marca el fin de la unidad entre el lenguaje y el mundo, entre la palabra y la cosa. Lo arbitrario del signo, como hoy decimos, era visto como calamidad y no como condición de posibilidad de la significación.

La arbitrariedad es por tanto un principio muy antiguo. Sólo se le han opuesto como naturales ciertos signos, interjecciones $u$ otras expresiones de estados anímicos. También para la filosofía escolástica la significación de las voces era ad placitum, lo que se suele entender como convencional, de institución 0 artificial; una concepción que pasa inclusive por los gramáticos de Port Royal, quienes se refieren a los signos calificándolos por la expresión "inventados por los hombres" y que no tienen ninguna semejanza con nuestros pensamientos. La filosofía iluminista, por su parte, mantiene el concepto de arbitrario, aunque sobre otras bases y otros objetivos.

M e referiré más abajo a Locke, pero antes es necesaria una referencia a otro filósofo de las Luces: Condilllac, quien postula una primera etapa de signos naturales en el sentido de un lenguaje cuyas señales respondían a la conformación corporal (movimientos corporales, gestos faciales, gritos), de ahí su condición natural. Pero este lenguaje, necesariamente limitado, es superado en la medida en que las necesidades materiales llevan a los hombres a crear signos artificiales (denominación que Condillac prefiere a la de "arbitrarios"), aunque siguiendo el modelo natural de aquel lenguaje primero. ${ }^{4}$

Las lenguas evolucionaron a partir de aquel estado primitivo por las diferentes necesidades de supervivencia, que incluyen el poder comunicarse. Así se van alejando del modelo primitivo en la medida que los grupos inventan nuevas ideas en su contacto con cada ambiente, también diferente. U na vez más, la cuestión de las diferencias entre lenguas es retirada del principio mítico-teológico y puesta en la actividad humana. Lo que queda del pasado es aquel origen natural y lo que obra la diferencia es la mayor o menor destreza para crear signos, que por cierto no es universal y varía según el modo de relación con el ambiente de cada grupo: los materiales son los mismos para todos los hombres;

4. Condillac distingue signos accidentales, los que alguna circunstancia particular ha enlazado con nuestras ideas; naturales, "gritos que la Naturaleza ha establecido para los sentimientos de gozo, de temor, etc.; y establecidos, "los que hemos escogido nosotros y solamente tienen una relación arbitraria con nuestras ideas". Ensayo..., p. 46. 
pero su utilización varía; de ahí la desigualdad que encontramos entre ellos. Q ueda así planteado el problema de la diversidad de las lenguas, dejada entonces en manos del trabajo humano con los signos, y queda así fundada la inseparabilidad entre lengua y cultura:

Recorramos ahora la superficie de la tierra y veremos que los conocimientosaumentan o disminuyen según si lasnecesidades son multiplicadaso limitadas. Reducidos casi a nada entre los salvajes, [losconocimientos] son plantasinformesqueno pueden crecer en un suelo ingrato, en el que carecen de cultura. En cambio, transplantados a las sociedades civiles, crecen, se extienden, seinjertan unos en otros, semultiplican detodas las maneras y varían susfrutosal infinito. Ensayo sobreel origen de losconocimientoshumanos (p.35)

Este pasaje deja ver una clave esencial de la antropología de las Luces: todos los hombres son naturalmente capaces de formarse nuevas ideas (recordemos que estas nos vienen de las percepciones) y de crear nuevos signos para ellas. Signos que impongan sistematicidad a la experiencia, y agrego: esta experiencia cultural establecerá a su vez sistematicidad en el material sígnico. Son las necesidades, que nacen de la confrontación con el ambiente, las que determinan que estas capacidades se desenvuelvan. D eahí el papel esencial dado a la educación en el pensamiento de la llustración.

En segundo lugar, las afirmaciones de Condillac parecen fundarse en la riqueza de observaciones y testimonios que durante los siglos XVII y XVIII muchos viajeros y exploradores pusieron en sus relatos sobre las costumbres y las lenguas de pueblos, para los europeos, exóticos. Esos relatos reflejan a menudo la perplejidad ante lo que se sentía como ausencia de sistema en los intercambios lingüísticos. Refiere Auroux (1994, p.114): "Sagard ve en el huron una lengua 'salvaje, casi sin reglas (... ) imperfecta' [... ] M onboddo concluirá que 'es imposible construir con ellos una gramática: esto es, reducirlos a alguna regla' [... ]". 5

En tercer lugar, la comparación del pensamiento primitivo con una planta no cultivada, la carencia de cultura de ciertas naciones, es un tópico queC ondillac sólo retoma y por el cual se opone la tierra dejada al crecimiento espontáneo a la tierra parcelada: la división del suelo es precondición para la producción diversificada. Volveré a este punto; por ahora, destaco del pasaje de Condillac también la idea de que la cultura se constituye y reafirma en relación a la diferencia. Esto se ve también en el modo como se forjan las identidades lingüísticas, en el temprano Renacimiento, con las dispersión del latín. Los

5. Se refiere a G. Sagard, Dictionnaire de la langue huronne, 1632; J. Burnett, conocido como Lord Monboddo, Of the Origin and Progress of Language, 1773. 
vernáculos europeos se afirman cada uno mirándose en el espejo de los otros. Las gramáticas particulares que así surgen son, además de instrumentos para aumentar las competencias, unificadoras de modos de decir que van juntos con los procesos de unificación política. Pero su particularismo también es señal de que ya no existe aquel imaginario de cultura universal que el latín ayudaba a sustentar. Aparece, con un alcance próximo al actual, la idea de la lengua extranjera. El mapa del mundo conocido se vuelve, en parte, también un mapa lingüístico. M apa político, con divisiones y fronteras. Fronteras simbólicas, las de las lenguas, dentro de las que se construyen identidades, y caminos simbólicos por los que se puede transitar, incluso sin salirse del mapa real: las gramáticas puede sustituir al aprendizaje por inmersión.

Podemos retomar ahora, con nuevos elementos el tema, mencionado antes, de la delimitación y su relación con la cultura. T. S. Eliot (1948) reproduce la entrada culture del diccionario 0 xford de 1483: "The setting of bounds; delimitation (rare)". La primera acepción nos lleva, por metonimia, a cultivo: poner vallas para plantar. Por su parte, la segunda acepción, aunque "rara", de "delimitación (de la tierra)" completa la primera; extendida al plano simbólico, atribuye a las lenguas la propiedad de delimitar, cada una de diversa manera, el mundo; así como el trabajo del suelo constituye - deslinda, ordena- lo social.

Y aquí debo remitirme a Saussure 0, más precisamente, a lo que ve en la propuesta saussereana de signo Jonathan Culler (1979, p.18-19):

El hecho dequedoslenguas, por ejemplo el inglésy el francés, operan $(. .$.$) con diferentes articulaciones 0$ distinciones conceptual esindica queesas divisionesno son naturales(subrayo) o necesariassino (... ) arbitrarias. U na lengua tendrá, sin duda, formas de nombrar masas de agua corriente, pero puede hacer susdistincionesconceptuales (... ) con cual quiera delas muchas manerasadisposición (extensión, velocidad deflujo, derechez o sinuosidad de éste, profundidad, navegabilidad, etc.). U na lengua puedeno sólo elegir arbitrariamentesus significantes, sino también dividir un espectro deposibilidades conceptuales decuantas manerasquiera.

Explica después Culler (p.19), según este principio, que los pares river / stream y fleuve / rivière (del inglés y francés, respectivamente) se construyen según diferentes criterios de oposición: la extensión, en el primer caso, el correr o no hacia el mar, en el segundo. Así, entrar en otra lengua nos pone en la situación de reconocer que no hay distinciones universales, necesarias, en la construcción de significados, sino maneras arbitrarias de ordenar el mundo. En el caso de las diferencias fónicas, esto es más evidente y no me detendré en ello. Pero en el plano conceptual, el que pretenda ver entre las lenguas sólo secuencias 
sonoras diferentes para nombrar "lo mismo" se encontrará con dificultades insolubles. ${ }^{6}$

También en estudios actuales, como un artículo célebre de Christine Revuz (1998) se ha señalado el conflicto entre las divisiones naturalizadas en la lengua materna y las de una segunda lengua. Revuz sitúa su reflexión en la instancia reveladora de la arbitrariedad que proporciona esa singular experiencia de aprendizaje: el contacto con otra lengua-cultura produce un movimiento reflejo que nos lleva a percatarnos de nuestro propio lugar. M ás allá de la "exultación o el desánimo" que eso produzca, lo que se da es el descentramiento de la propia lengua:

\begin{abstract}
Q ue haya en ruso sólo una palabra para decir brazo y pierna, queel sol seafemenino en al emán, quelosingleses digan "yo soy frío"(... ) es desconcertante. Lo quesehaceañicosen el contacto con la lengua extranjera es lailusión de que existeun punto de vistaúnico sobre las cosas (... ) la ilusión de adecuación de la palabra y la cosa. Por la intermediación dela lengua extranjera seesbozael desplazamiento delo real dela lengua. Lo arbitrario del signo setorna una realidad tangible... (p. 223).
\end{abstract}

Sumemos a esto que, en tanto lengua, toda lengua extranjera, aun en su unidad estructural, alberga variación y que su aprendizaje impone un contacto no con una única forma diferente de ver e interpretar el mundo, sino con muchas y diversas, es decir, con una nueva, segunda diversidad. Se tratará, entonces, de hacer visible al aprendiz de idiomas lo que también le ocurre en la propia lengua, aunque de modo quizá menos, o nada, perceptible para él. Cultura es un modo de nombrar un mundo clasificado de una cierta manera, de nombrarlo y predicar sobre él, de interactuar con otros que comparten esas clasificaciones. Vemos el mundo desde una lengua y desde un dialecto: no hay posiciones ubicuas, hay identidades.

Siguiendo con la figura de la delimitación (nombrar la condición del proceso, colocar vallas, en lugar del proceso mismo, plantar), ella permite distinguir, junto con culturas, modos de producción, latifundios, vastedades más o menos improductivas, frente a parcelas, obstáculos para la producción en gran escala, etc. Esto también deja distinguir primero "sociedades", sistemas económicos, formas de propiedad; es decir: nos permite ver la base material de la cultura y así limpiar ese concepto de su vaguedad, de su lastre idealista; y, segundo, ver la cultura como conjunto de conceptualizaciones determinadas por formas de

6. Incluyo al final un comentario de Benveniste sobre Saussure que se relaciona con este punto. 
sociedad. Por otra parte, esto hace aparecer el factor ideológico, en general ausente 0 descuidado en propuestas de pedagogía de idiomas, en las que, sin embargo, a veces es posible leer ideología donde dice simplemente cultura.

M e propongo ahora - sin abandonar la línea conductora de la delimitación del mundo- señalar la contribución del empirismo (Locke) y el sensualismo (Condillac) a la idea de cultura basada en el funcionamiento del lenguaje. Esto es, mostrar de qué manera las lenguas son responsables del efecto cultura (y la cultura, podemos decir hoy, es simultáneamente responsable del efecto lengua).

Condillac, como anticipé, ve al lenguaje de signos artificiales como superación del lenguaje natural de gestos y gritos ("lenguaje de acción"). Ligado éste al complejo simultáneo de las sensaciones, al que busca representar en toda su simultaneidad, el primero, en cambio, por su carácter lineal, transfiere al pensamiento su propia sucesividad: las lenguas son métodos de análisis del pensamiento en tanto permiten formar ideas distintas a partir del todo confuso de las ideas-sensaciones. Las lenguas dan al pensamiento su discursividad.

Es su predecesor Locke quien introduce la tesis de la naturaleza lingüística del pensamiento. Esta debe entenderse como refutación de las teorías escolásticas, que atribuyen a la mente (modi intelligendi) la posibilidad de captar un mundo jerárquicamente ordenado en géneros y especies: un mundo que responde al ordenamiento perfecto de un Creador supremo. Para Locke no hay ideas universales. Lo que surge de la acción de los sentidos sobre lo real son ideas simples de las cosas particulares: "todas las cosas que existen son particulares". Las ideas complejas son la reunión de cualidades percibidas en un mismo objeto. Y son estas colecciones de cualidades lo que nombran las palabras. Los nombres, sin embargo, a excepción de los nombres propios, son términos generales, se aplican a todo particular. "Los hombres - dice Locke - nunca han intentado dar nombres a cada oveja de sus rebaños, o a cada cuervo que vuela sobre sus cabezas, y mucho menos llamar por un nombre particular a cada hoja de las plantas, a cada grano de arena que vieran." (Ensayo, p. 398).

"La elección que se realiza en la constitución de los nombres nunca es estable ni exhaustiva", dice Lia Formigari, y agrega que dicha "elección"

nunca se apoya en la intuición de la esencia real de la cosa; al contrario, el campo de significación es cada vez ampliado o restringido o variado según las exigencias funcionales de la comunicación. El significado esen sumaunainformación sobre los objetos, cada vez delimitado por el signo, y que nunca es homólogo deesos mismos objetos. (1992, p.447) ${ }^{7}$

7. Agradezco a Luci Banks Leite por señalarme la importancia de esta lectura. 
Con Locke se da, entonces, una verdadera revolución, que se produce "en el marco de la crítica a la filosofía escolástica", y "esa refutación conduce a subrayar el poder del lenguaje en la clasificación del mundo cognoscible, a subrayar su independencia ante los realia y así, en última instancia, a sostener lo arbitrario del signo lingüístico" (p. 443). La autora trae a consideración, junto a la filosofía de John Locke, la física de Robert Boyle. Ambos tratan la cuestión de las cualidades de las cosas y del proceso por el que el espíritu clasifica lo real. $\mathrm{H}$ asta aquí veíamos al lenguaje delimitar el mundo, veamos ahora la base de ese proceso, el modo cómo el pensamiento construye clases.

Toda esta crítica, en el siglo XVII, parte de una posición escéptica: no tenemos acceso a las esencias últimas, si pudiésemos conocerlas, tendríamos nombres fijos para ellas y las lenguas serían lo que Saussure nos dice que no son, nomenclaturas. Citando a Boyle, contemporáneo de Locke, Formigari, muestra la visión de un científico sobre el conocimiento humano de la materia:

no conocemos de los cuerposmás quesus cualidades sensibles, y no podemos pues distinguir los génerosy lasespeciesmásque en base a una clasificación empírica de las cosas (Boyle). Procedemos, entonces, en basea 'un conglomerado deaccidentes quela mayoría de los hombres, por una especie deconvención (... ) considera necesarias o suficientes para que una partedela materia universal pertenezca a tal o cual género o especie de cuerpos naturales' (Robert Boyle, The O rigin of Forms and Q ualities, according to Corpuscular Philosophy, 1666).

y señala la vigencia de esa visión al ser traducida a términos lingüísticos:

esteargumento epistemológico implicaba una (... ) revolución de la teoría semiótica: en efecto (... ) si las clases de objetos designadas por los nombres no son coleccionesfundadas en la unidad natural o real delosindividuos, sino conjuntos arbitrarios de cualidades, entonces los nombres que designan estas colecciones serán tanto másarbitrarios. Serán inclusiveel único elemento que reúne individuos en colecciones. (Formigari, 1992, p.444)

La revolución semiótica implícita en esta nueva epistemología será llevada adelante por Locke en su Ensayo sobre el entendimiento humano (1690). Podría decirse que Locke inaugura la semiótica al explicar la cuestión de las ideas generales. Las cosas no están presentes por sí mismas en el espíritu; hay una instancia intermedia: el signo o la representación de la cosa. El signo se caracteriza por la generalidad, ya que es la reunión de varios particulares. El signo debe a aplicarse a la experiencia, que, sin embargo, es sólo de las cosas particulares. ¿Cómo es esto posible? Gracias al proceso de abstracción, que nos 
da ideas separadas de las circunstancias de la existencia real (lugar, tiempo, etc.); estas son las ideas generales, y los nombres comunes o términos generales son la representación de esas ideas generales. I deas generales y nombres son entonces la reunión de colecciones de cualidades. D e nuevo la pregunta: ¿cómo es que los términos generales pueden representar las cosas, que siempre son particulares? Justamente porque las ideas abstractas sirven de mediadoras entre los nombres y las cosas. Esas ideas abstractas son llamadas por Locke, por eso mismo, esencias nominales: existen porque las esencias reales son incognoscibles.

La abstracción es menor en el caso de las ideas simples, provenientes de sensaciones primarias, el color, el calor, la rugosidad... En el caso de sustancias, un animal, una planta, la abstracción es mayor. Y es máxima en el caso de los términos morales y jurídicos. La arbitrariedad también es creciente en la misma escala, ya que la selección de las cualidades de la sustancia es el trabajo de la subjetividad y no una lectura precisa de lo natural. Veamos los casos que menciona Locke, que nos recordarán los de Sausssure y los de Culler, dados arriba. Por ejemplo, en la naturaleza no hay un vínculo más específico entre la idea de matar y la de hombre que entre aquella y la de ganado; sin embargo, sólo hay denominación específica del acto (homicidio) en el primer caso.

Este último ejemplo muestra que el nombre abstracto homicidio no tiene correlación en la práctica de matar ganado, para la cual no habría en inglés un nombre específico. La existencia del nombre homicidio se debe a la necesidad de calificar moralmente un acto; la inexistencia de denominación específica para la acción de matar animales indicaría, entonces, que esa acción no está moralmente señalada; lo que se entiende en sociedades donde esa acción corresponde a una necesidad práctica de alimentarse 0 vestirse. ${ }^{8}$

Eso significaquelaclasificación delas cosasy losacontecimientos, y su denominación respectiva, sedan en virtud defines prácticos, quees determinada por las necesidades de la comunicación y dictadas por factoresdecostumbres, detradición, dehábito; en suma, por factoresculturales. (Formigari, 1992, p.446)

$Y$ aunque esto es claro en términos que no tienen una referencia concreta, el mismo principio rige, para Locke, en la designación de las cosas. Comentando un pasaje del Ensayo de Locke, Formigari resalta (1992, p.446.):

N o sólo se aplica esto a términos morales, sino también a la designación de las cosas: diferenciamos por su nombreal agua

8. Un filólogo podría objetar que sí hay este tipo de designaciones en otras lenguas, acaso schlachten en alemán correspondería al término ausente en inglés, pero esto corroboraría la tesis "culturalista" de Locke. 
fluentey al agua congelada, pero no sehaceesa distinción entre el oro sólido y el fundido en el crisol del joyero. Además, un mismo nombrepuededesignar paraalgunosuna colección más amplia, para otrosmás restricta, deideas simples: paraun niño, basta un color amarillo brillantepara dar la idea deoro, otros le agregan la maleabilidad, lafusibilidad, el peso específico; y otros, incluso, otras cualidades.

Aquello que nombramos como lo arbitrario del signo no es "la simple indiferencia del sonido en relación a la cosa". Es una consecuencia del proceder semiótico del espíritu, del modo como forma los conceptos: delimitando y clasificando arbitrariamente lo real en una actividad en la que intervienen, como se dijo, factores de moral, de hábitos, etc.

Resumiendo: las ideas generales se interponen entre el sujeto y lo real, y los nombres son las expresiones de esas ideas generales. El conjunto de cualidades percibidas con que esas ideas generales se forman es variable y fluctuante, no natural sino arbitrario. Así lo vimos en los ejemplos de fleuve frente a rivière, de river frente a stream; como también en los ejemplos de Locke, más los que aparecen en el cap. IV, parte II, del Curso de Saussure. El entender la misma cosa bajo un mismo nombre, es debido a un consenso comunitario: el espacio de ese consenso es el espacio de una cultura. No hay, pues, cultura sin lengua porque somos incapaces de pensar sin ideas generales, que son los signos de las cosas y que están determinadas por los atributos que cada grupo, comunidad 0 sociedad convenga reunir en ellas.

En la elaboración de este texto he reunido diversas nociones dispersas en siglos y tendencias. H e mostrado también algunos ejemplos de esa preocupación que lleva a considerar los aspectos discursivos presentes en las prácticas de hablar y aprender otra lengua. Esto me llevó a buscar en la historia algunas ideas en torno a la significación y a hacerlas presentes. D ecir significación a la luz de los autores tratados es, como decía, hablar de arbitrariedad, no solamente en cuanto a la relación de los componentes del signo. Así, en el movimiento de la historia, la visión saussereana aparece enriquecida en las páginas de Culler y quizá deslucida al ser confrontada con la semiótica de Locke, en la cual la arbitrariedad se da de un modo radical al ser referida, más que al principio del significado y el significante, a la condición fundamental de la significación: la formación de ideas generales. En esa construcción de la generalidad está comprometida la convención, en ésta lo social, y en esto, a su vez, lo cultural. La significación, así vista como praxis humana es dinámica, móvil y nunca exhaustiva. 
Ahora bien, la filosofía de las Luces privilegió los nomina, en los que se muestra con más inmediatez la relación entre el lenguaje y las sustancias (nombre sustantivo) y sus accidentes (nombre adjetivo). Pero es posible, sin alterar aquellos principios de la significación, incorporar el saber lingüístico actual. Formigari (1992, p.446) amplía de este modo el principio de la formación de los nomina:

Losfactores prácticos que entran en la elección realizada en el acto de habla son numerosos y variables: los usos lingüísticos corrientes; las características dela lengua histórica queseutilice; las necesidades de la comunicación que llevan a poner en evidenciaciertosaspectos delas cosas de quesehablay no otros; los hábitoso las convenciones estilísticas ligadas a los diversos tipos decomunicación (...); los hábitos lingüísticosligadosala pertenencia cultural 0 a la clasesocial de losinterlocutores, etc. (... ) (Formigari, 1992, p.446).

Valida así el cuadro de la semiótica empirista extendiéndolo a fenómenos estudiados después por la pragmática, la gramática funcionalista y la sociolingüística. D ebo, por mi parte, agregar la sintaxis, que la autora omite, y que, consecuentemente con esta imbricación de lengua y cultura, resurge despojada de todo universalismo.

Para concluir quisiera hacer una referencia a Benveniste (1976) en sus precisiones sobre la arbitrariedad del signo en Saussure y a su propia propuesta: propone remplazar ese principio por el de necesidad y reservar lo arbitrario para la relación entre el signo y la cosa. Las dos partes del signo mantienen una relación "consustancial": existe entre significado y significante "una simbiosis tan estrecha que el concepto 'boeuf' es como el alma de la imagen acústica "böf", y es esa necesidad lo que "asegura la unidad estructural del signo". Y prosigue: "lo que es arbitrario es que tal signo, y no tal otro, sea aplicado a tal elemento de la realidad" (p.52). Atribuye el hecho de que Saussure sitúe la arbitrariedad en el interior del signo, y no entre éste y la "cosa", al sustento de la tesis de que la lengua es forma; lo que lo lleva a dejar la sustancia "fuera de la comprensión del signo".

Benveniste ve, además, una contradicción a ese antisustancialismo saussereano en el propio texto del Curso. Según Benveniste (1976), cuando Saussure aduce, a favor de la arbitrariedad, que "el significado 'boeuf' tiene por significante b-ö-f de un lado de la frontera y o-k-s (0 chs) del otro" (p.52), introduce "un tercer término, que no estaba comprendido en la definición inicial" y "este tercer término es la cosa misma, la realidad" (p.50). Benveniste 
entiende que ese tercero aludido es un segmento de "realidad concreta y sustancial" introducido "inconsciente y subrepticiamente" en la teoría; y explicita: "[Saussure] se refiere a pesar suyo al hecho de que estos dos términos ('boeuf' y '0 chs') se aplican a la misma realidad". (p.50).

Pero cuando un segmento de realidad es traído adentro de la significación del signo, ¿conserva su estatuto de "real"? Siguiendo el ejemplo saussureano, cuando la sustancia buey es designada como boeuf de un lado y como 0 chs del otro lado de la frontera (lingüística), ¿sigue siendo la misma entidad? En términos más simples: ¿es el mismo buey? Creo que Locke, dados su ejemplos, diría que no. Y esto se ve más claro en otros ejemplos del mismo capítulo del Curso: sea el caso de significado soeur (hermana) o del de cualquier otro término de parentesco: ¿diríamos que es el mismo segmento de lo real? Aquí volvemos a encontrar las delimitaciones del mundo que, aunadas, la lengua y la cultura construyen.

Benveniste atribuye el desliz saussureano a un resto del "pensamiento histórico y relativista de fines del siglo XIX", a la "inteligencia comparatista", en la que "de la universal desemejanza se concluye la universal contingencia" (p.51-52). ¿Y no es así cuando se ve a la lengua y a la cultura construyendo juntas, en reciprocidad la clasificación del mundo?

\section{Referencias bibliográficas}

AU RO U X, Sylvain (ed.). H istoiredesidéeslinguistiques, tomo 2. Lieja: M ardaga, 1992, 683 p. AU RO U X, Sylvain. La révolution technologiquedela grammatisation. Lieja: M ardaga, 1994, $216 \mathrm{p}$.

BEN VEN IST E, Émile. Problemas de lingüística general, cap. IV, La naturaleza del signo lingüístico. 6.ed. M éxico: Siglo XXI. 1976, 218 p.

CON D ILLAC, Étienne. Bonnot de. Ensayo sobreleorigen delos conocimientoshumanos, M adrid: Tecnos, 1999, $291 \mathrm{p}$.

CULLER, Jonathan. AsidéiasdeSaussure. São Paulo: Cultrix, 1979, 105 p.

ELIO T, T homas Stearns. N otaspara la definición dela cultura. Buenos Aires: Emecé, 1949, $189 \mathrm{p}$.

FO RM IG ARI, Lia. Le langage et la pensée. In: AU RO U X, Sylvain (ed.). H istoi re desidées linguistiques, tomo 2. Lieja: M ardaga, 1992, 683 p.

LO CKE, John. Ensayo sobreel entendimiento humano. 2.ed. M éxico: FCE, 1999, 753 p.

N IZAN , Paul. Lesmatérialistesdel'antiquité. París: M aspero. 1971, 136 p.

REVU Z, Christine. A língua estrangei ra entreo desejo deum outro lugar eo risco do exílio. In: 
Pro-Posições, v. 19, n. 1 (55) - jan./abr. 2008

SI G N O RIN I, Inês (ed.). Linguagem ei dentidade: Elementos para uma discussão no campo aplicado. Campinas: M ercado das Letras. 1998, 384 p.

Recebido em 06 deagosto de2007 eaprovado em 23 denovembro de2007. 